\title{
CALIDAD EN EL SERVICIO EN LA EDUCACIÓN A DISTANCIA. UNA PERSPECTIVA DESDE MÉXICO
}

\author{
(QUALITY OF SERVICE IN HIGHER DISTANCE EDUCATION. A PERSPECTIVE FROM MEXICO)
}

\author{
Elia Marúm-Espinosa
}

Universidad de Guadalajara (México)

\section{RESUMEN}

¿Qué significa una buena educación o calidad en el servicio en la Educación a Distancia? Debería significar lo mismo que para la educación presencial. La calidad de la educación institucional no depende de la modalidad educativa, sino de la calidad de los procesos que se viven y aprenden. En México no contamos aún con normatividad que reglamente el funcionamiento de los llamados "nuevos proveedores de educación”, ya que en el caso de la educación a distancia, muchos de ellos no requieren el Reconocimiento de Validez Oficial de Estudios (REVOE). Las y los docentes mexicanos se identifican con conceptos "modernos" de calidad, en tanto que la de directivos y gestores lo fue para los conceptos "tradicionales" de calidad. Las concepciones de calidad que tienen estos dos actores y las acciones que de estas percepciones se derivan, apuntan de manera divergente hacia la consecución de una buena educación.

Palabras clave: calidad educativa, calidad en la educación a distancia, calidad percepciones de los actores, docentes y educación a distancia de calidad.

\begin{abstract}
What constitutes good education or quality service in distance higher education? It should be the same as for conventional education. The quality of institutional education does not depend on the mode of delivery; rather, it refers to the quality of the processes that are lived and learned.

In Mexico the so-called "new educational providers" have yet to be regulated. Many of the socalled new providers of higher distance education are not required to obtain official recognition of studies (REVOE). While higher education teachers identify with "modern" concepts of quality, their administrators identify with "traditional" concepts of quality. The conceptions of quality of these two groups and the actions that reflect these conceptions point to divergent ways to attain good education.
\end{abstract}

Keywords: quality education, quality of distance higher education, quality and stakeholders, faculty and quality distance learning. 
Si bien México tiene un reto enorme para lograr en educación superior la cobertura mínima que puede garantizarle la sustentabilidad de su desarrollo económico, social y ambiental -que desde 1994 UNESCO recomendó y la Organización para la Cooperación y el Desarrollo Económicos (OCDE) retomó, fuera entre 40 y 50\% del total de jóvenes en edad de demandarla-, ya que está entre 10 y 20 puntos porcentuales por debajo de este mínimo, es hasta ahora que ha comenzado a preocuparse por el reto de la cantidad, puesto que en las últimas dos décadas ha prevalecido, como preocupación fundamental de las políticas públicas e institucionales, el reto de la calidad.

"En la actualidad, el conocimiento ha tenido un papel protagónico en el desarrollo de las naciones y empresas. La aportación de las universidades ha sido fundamental, sobre todo en los países desarrollados, ya que gracias a las investigaciones que allí se realizan y a la formación de profesionistas, han logado mayores niveles de competitividad y bienestar para su población [...] pero en la mayoría de los países emergentes no hay una visión clara de cómo las universidades pueden ayudar a lograr mayores niveles de competitividad" (Nava et al., 2010), ni cómo incorporar, de manera contundente y eficiente, los ambientes de aprendizaje no presenciales o presenciales enriquecidos por las tecnologías de la información y la comunicación (TICs) a sus modelos educativo y académico.

Estamos en un cambio de época, en una transición que nos lleva de la sociedad industrial a las sociedades del conocimiento (UNESCO, 2005 y World Bank, 2002), sociedades que han erradicado la pobreza, que han asegurado la sustentabilidad de su desarrollo y un nivel adecuado de vida para toda la población, porque han incorporado el conocimiento a sus procesos productivos y sociales, y con ello se han ubicado en el plano de una fuerte competitividad económica basada en las actividades que agregan alto valor económico y social porque integran conocimiento de gran calidad, minimizando las actividades tradicionales que lo hacen débilmente.

Es aquí donde la educación y sus nuevas modalidades ubican su importancia, reconociendo las condiciones históricas de México en un estrato de desarrollo menor que el de los llamados países desarrollados. "Un modelo de sociedad del conocimiento adecuado para México debe promover la supresión de las asimetrías socioeconómicas, garantizar una amplia inversión en educación, en ciencia y tecnología, así como fomentar el crecimiento y el aprovechamiento público de los conocimientos, saberes y técnicas tradicionales que se cultivan en muchas regiones del país, las cuales constituyen fuentes potenciales para la innovación y el desarrollo" (Olivé, León y Ricardo Sandoval, 2007), para lo cual la virtualidad y en general la 
educación a distancia constituyen un poderoso medio para utilizar y difundir el conocimiento que se requiere en procesos de formación educativa y para generar opciones de ampliación de la matricula y flexibilidad curricular.

La educación a distancia significa una ruptura que ha ocasionado "el cambio de los espacios reales de aprendizaje por los espacios virtuales. No era previsto, mucho menos deseado o planeado, por ningún pedagogo. Este cambio significa que los maestros y estudiantes ahora se ven expuestos a una situación que tiene una estructura completamente diferente y que ofrece una variedad de oportunidades nuevas" (Peter, 2002). Pero, por ello, "las modalidades virtuales se constituyen, asimismo en una significativa complementación de la educación superior, por lo que será necesario reelaborar los modelos educativos vigentes en una y otra modalidad de manera de poder integrarlos adecuadamente" (Fernández, 2010), sólo que la gran mayoría de los actuales modelos educativo y académico de las instituciones de educación superior mexicanas no han realizado esta integración y con ello se han diluido las sinergias que podrían generarse.

\section{¿CALIDAD EN LA EDUCACIÓN O UNA BUENA EDUCACIÓN?}

El concepto calidad proviene del ambiente empresarial, de los esquemas de administración de la calidad total (Total Quality Management, propuesto entre otros por Edwards Demming, 1989) y ha penetrado fuertemente en la educación hasta casi haber cobrado carta de naturalización. Sin embargo, "debemos estar alertas a algunas desviaciones de la educación actual que impiden el logro de una buena educación: la sobre valorización de lo económico, la competitividad a ultranza y el culto a la excelencia, también proyección de las manías de "calidad total" que han invadido el mundo de los negocios y que siguen siendo la base sobre la cual se construyen las políticas públicas y las políticas institucionales de este nivel educativo" (Latapí, 2008). Por eso, retomando la propuesta de Latapí (2008), es indispensable comenzar a referirnos a una buena educación en lugar de educación de calidad, con lo que desde el lenguaje abonaremos a separar a la educación de la lógica empresarial y de mercado.

Pero ¿̇qué es una buena Educación? De acuerdo con Latapí (2008), es la que busca como resultado e impulsa cuatro rasgos característicos que son inseparables:

- El carácter (congruencia entre pensar y obrar), convicciones claras y firmes y un sentido de finalidad que engloba y afecta todo esto que llamamos nuestras vidas; 
- La inteligencia que se desarrolla a través de y conjuntamente con el lenguaje para saber comunicarse y convencer, y de haber asimilado un sentido de la historia, una interpretación consciente del pasado que permita entender mejor el presente y debe ser educada (capacidades de abstracción, raciocinio lógico, análisis, síntesis, relación, inducción, deducción o aprender a pensar y aprender a aprender);

- Los sentimientos, casi olvidados por el racionalismo pedagógico prevaleciente, tienen elementos como el cultivo de la imaginación y la creatividad, el desarrollo de la intuición, la modulación de la sensibilidad y la educación para la compasión;

- La libertad, educar para la libertad posible y para la libertad responsable, con el esclarecimiento de los propios valores la formación de normas para discernir el bien y el mal.

Esto es, un proceso de transformación y formación integral del individuo, tal como lo plantea el Artículo $3^{\circ}$. Constitucional, y o un proceso reducido al cumplimiento de indicadores por si mismos sin que estos tengan una repercusión en la transformación del trabajo cotidiano y en los valores y el espíritu que lo inspira.

\section{UNA BUENA EDUCACIÓN, UNA EDUCACIÓN DE CALIDAD}

Mas allá de buscar una definición formal que no resolvería la complejidad de la conceptualización de la calidad educativa, ¿qué significa calidad en el servicio en la Educación a Distancia o qué significa una buena educación a distancia?, suponiendo que ya hemos acotado lo que es la educación a distancia y que en esta delimitación no la hemos reducido sólo a la educación virtual (o e-learning), aunque esta sea un componente fuerte de esta modalidad educativa. Desde la perspectiva de las instituciones de educación superior como instituciones formadoras de individuos para una vida social y productiva plena y responsable, debería significar lo mismo que para la educación presencial. "La calidad de la educación institucional no depende de la modalidad educativa, sino de la calidad de los procesos que se viven y aprenden, la cual está condicionada, fundamentalmente, por el personal académico, el currículum, el apoyo a estudiantes, los recursos de información y conocimientos, y su accesibilidad y procedimientos de evaluación válidos y confiables para la institución, la sociedad y el propio estudiante" (Moreno 2007).

Proporcionar una buena educación es la razón de ser del proceso educativo, "la educación universitaria es, esencialmente, un proceso cualitativo. Pero si estamos seriamente interesados en evaluar su calidad tenemos que poner en el centro el 
carácter, el contenido y la orientación de esta educación, comprendida como un proceso de transformación de los individuos y de la sociedad, [...] ubicar la calidad sólo en los records de los productos de investigación, o las tasas de empleo de los egresados no representa el problema principal a abordar" (Didriksson, 2003). Por tanto, mejorar y evaluar la calidad educativa debe hacerse siempre, en todo momento y en todos los ambientes de aprendizaje que constituyan el proceso formativo, donde se motive, valore y aprecie al estudiantado; donde se haya cambiado el proceso docente de enseñar contenidos y lograr objetivos de un programa de un curso o espacio de formación dentro del plan de estudios, seguido conductistamente, a generar procesos de aprendizaje cualificados, acordes con la naturaleza de los aprendizajes y competencias que los hagan aplicable; donde se haya transformado la práctica docente y de gestión, no importa la modalidad, en una práctica motivante y centrada en el aprendizaje de las y los estudiantes, con estrategias didácticas múltiples que los involucren en cada una de las tareas a realizar dentro y fuera del aula; con recursos institucionales que aseguren la motivación del profesorado y con recursos didácticos múltiples que propicien la motivación del estudiantado, para innovar los procesos de mediación profesor(a)-alumno(a) y adecuarlos a la modalidad educativa (abierta, a distancia, virtual, mixta o presencial), reconociendo el contexto y las condiciones de quienes reciben la educación, esto es, interesándose genuinamente por conocer y comprender las características y condiciones que enfrenta cada estudiante para realizar su formación, así como conocer y comprender sus capacidades, vocaciones, intereses y sentimientos.

\section{CALIDAD EN LA EDUCACIÓN A DISTANCIA O UNA BUENA EDUCACIÓN A DISTANCIA}

Una buena educación a distancia no se limita a la simple prestación de un servicio, ni puede evaluarse su calidad como se hace con la generación de servicios, sino que, al igual que cualquier otra modalidad educativa, es una acción de transformación humana, con toda la complejidad que ello implica, de ahí que gestionar la calidad educativa, asegurar una buena educación, no sea fácil ni se pueda limitar a manuales de procedimientos o procesos estandarizados, como puede suceder en la prestación de otro tipo de servicios, ni se limite al dictado de clases, al cumplimiento de los contenidos académicos del programa de los cursos, a contar con aulas equipadas, etc., sino que tiene que ver con la realización de la docencia en múltiples espacios de aprendizaje y con múltiples actividades formativa, desde el acompañamiento permanente (tutorías), las prácticas permanentes, hasta el conocimiento de las individualidades y condiciones de cada estudiante, y la generación de lazos afectivos de valoración y respeto, esto es, la interacción humana. 
A pesar de esta complejidad, existen esfuerzos por contribuir a orientar la gestión de la educación a distancia por la ruta de la buena educación. Se han propuesto indicadores para evaluar la calidad en el servicio en educación virtual y a distancia, entre ellos, Gayol y Seidensticker (2004) señalan los siguientes, a las que hemos hecho algunas puntualizaciones:

- Acceso, consistente en el diseño interactivo, transparencia y estabilidad tecnológica, y facilidades para las características de la población atendida, tanto en edad y capacidades, como en condiciones económicas, geográficas y sociales.

- Diseño pedagógico, para asegurar la calidad del contenido, el seguimiento permanente del proyecto, su alta eficiencia terminal. Seguramente las autoras se refieren a que la educación a distancia no reproduzca las limitaciones y obsolescencias de la educación presencial, generándose lo que pedagógica y didácticamente hemos llamado “tecnologizar la obsolescencia”.

- Diseño visual, que permita calidad de la instrucción, una eficiente relación costo/ efectividad y adecuados, suficientes y oportunos servicios de soporte (apoyo). Sin duda, esto requerirá el apoyo de personal especializado en diseño de ambientes virtuales, ya que la inmensa mayoría del profesorado no tenemos esta capacidad en nuestro perfil y no sería rentable académicamente utilizar nuestro tiempo en adquirirla, habiendo ya especialistas que nos complementan.

- Diseño instruccional adecuado al contexto sociocultural, a la intensidad de uso que garantice el impacto buscado, considerando lo planteado en el primer punto de este listado.

- Viabilidad financiera que asegure y garantice la capacidad en el largo plazo de mantener la modalidad educativa ofrecida. Cabe aclarar que en las instituciones de educación superior mexicanas no existen o no son conocidos los estudios actuariales para asegurar la viabilidad financiera que mantenga la actualización y funcionamiento de la introducción de tecnologías para el aprendizaje sustentadas en Internet o en alternativas virtuales o mediadas por computadoras, lo que ha ocasionado que se inviertan importantes sumas de dinero en proyectos a los que no se les ha calculado su sustentabilidad en el mediano y largo plazos, lo cual es inaceptable considerando las condiciones de restricción presupuestal que enfrenta la inmensa mayoría de ellas, ante las condiciones de pobreza y la enorme cantidad de necesidades no satisfechas de la población mexicana.

- Complementariedad y tránsito entre modalidades, de manera que no existan limitaciones normativas, administrativas o de otro tipo para que se pueda 
complementar la modalidad presencial con las modalidades semi presencial, abierta y a distancia, y se facilite el tránsito entre ellas.

Todo ello aderezado de la calidez humana y el genuino interés por la transformación positiva de las y los alumnos, en un compromiso de formadores, directivos y gestores para que puedan generarse las condiciones necesarias en el trabajo cotidiano. La gestión de la educación a distancia, al igual que para cualquier otra modalidad resulta crucial para lograr una buena educación, y las condiciones y funcionamiento de la gestión de un país que no ha alcanzado el desarrollo y que tiene una fuerte dependencia científica y tecnológica del conocimiento generado en otras realidades, y acertadamente diagnosticado en el Programa Especial de Ciencia y Tecnología 2008-2012 (Consejo Nacional de Ciencia y Tecnología, 2008), hacen que sus marcos teórico referenciales para la gestión no consideren opciones autóctonas de desarrollo tecnológico y que de manera acrítica y a veces hasta inercial se adopten modelos y técnicas educativas que no son las respuestas adecuadas a la condiciones específicas de la realidad mexicana.

El norteamericano Guy Neave (2009) lo reconoce al afirmar que "los directivos y académicos en México están deslumbrados por la educación superior y el pensamiento Norteamericano. Figuras como Clark Kerr, Martin Trow, Burton Clark, son quienes han conformado los marcos de referencia para el análisis de los sistemas de educación superior" y por tanto, podemos extender esta opinión a la educación a distancia donde Börje Holmberg, Michael G. Moore, Otto Peters, entre otros, son quienes más han influido, minimizándose el desarrollo de ciencia autóctona y la búsqueda de tecnología propia y adecuada a las estrecheces presupuestarias de los escasos recursos públicos y las condiciones estructurales de subdesarrollo.

\section{NORMATIVIDAD Y REGULACIÓN PARA LA EDUCACIÓN A DISTANCIA}

Hemos dicho que la virtualidad es un elemento importante de la educación a distancia y es "el principal instrumento para proveer de programas educativos transnacionales, por lo que agrega una complejidad adicional a las ya difíciles propuestas para su regulación" (Fernández, 2010). La complejidad que presenta esta modalidad educativa, se traslada entonces a la normatividad nacional e institucional que se requiere para asegurar su buen funcionamiento y garantizar que cumpla las expectativas sociales que genera. 
"A fin de lograr una adecuada regulación y evaluación de la educación superior virtual, en especial por sus fuertes efectos sobre la internacionalización de la educación, es necesario analizar a la educación virtual tanto desde lo institucional como en relación con programas y carreras, en lo conceptual y metodológico. Los países e instituciones proveedoras deberán controlar seriamente la calidad de los programas que producen y exportan, y hacerlo en un marco de respeto a las normas vigentes en los países importadores, trabajando en conjunto con las instituciones educativas locales" (Fernández, 2007).

En México no contamos aún con una Ley de Educación Superior, ni mucho menos con una Ley de Educación Superior, Ciencia y Tecnología, aunque para esto último si existe una Ley. Tampoco contamos con normatividad que reglamente el funcionamiento de los llamados "nuevos proveedores de educación", ya que en el caso de la educación a distancia, muchos de ellos no requieren el Reconocimiento de Validez Oficial de Estudios (REVOE) por encontrarse en otro país o por ofrecer sus servicios en Internet, casos donde las leyes mexicanas tienen serios vacíos pendientes de cubrirse.

Un ejemplo de los elementos que se incluyen en la normatividad de la educación a distancia para asegurar a la sociedad una buena educación, una educación de calidad, se encuentra en la Ley de Educación Superior Ciencia y Tecnología de la República Dominicana donde destacan los siguientes elementos de esta normatividad: a) la incorporación y la aplicación de las nuevas tecnologías informáticas, requiere nuevos planteamientos regulatorios que sustenten tanto la planificación, la administración y la operatividad como lo pedagógico y evaluativo del quehacer formativo; b) se necesita que el sistema nacional de Educación Superior, Ciencia y Tecnología (SESCYT) sea abierto y flexible; c) asegurar mecanismos para facilitar la transferencia de los estudiante entre las diferentes instituciones y modalidades; d) impulsar, fortalecer y consolidar modalidades educativas a distancia en las instituciones del SESCYT, en correspondencia con su finalidad, misión, valores y objetivos; e) regular el funcionamiento de la modalidad de educación superior a distancia en todas las instituciones publicas y privada.

De igual forma establece que toda institución de educación superior a distancia que se establezca tiene la obligación de enunciar sus fines y sus principios, siempre en correspondencia con el marco y los alcances de la finalidad de la educación superior antes expresada, consignada en la Ley 139 - 01, de Educación Superior, Ciencia y Tecnología y este Reglamento, y que las instituciones extranjeras que deseen instalarse en República Dominicana e impartir programas en la modalidad 
a distancia deberán cumplir con la los programas de educación a distancia que se ofrecen en las instituciones de educación superior, deben cumplir los requerimientos de planificación, organización, gestión, desarrollo y evaluación que exige esta modalidad educativa, a fin de constituir un sistema pedagógico con sus componentes integrados en relación con sus objetivos.

Esta normatividad coincide con los elementos que se han dado para considerar lo que es una buena educación a distancia, dentro de lo que se ha conceptualizado como calidad en el servicio de la educación a distancia.

\section{PERCEPCIONES DE LA CALIDAD DE LA EDUCACIÓN SUPERIOR DE DOCENTES Y GESTORES MEXICANOS}

Pero en realidad ¿cómo perciben la calidad educativa quienes son docentes universitarios en México?, ¿cómo lo hacen quienes son gestores de la educación superior? Hasta ahora se ha escrito mucho sobre el deber ser, "la doctrina" de calidad educativa, sin que haya mucha evidencia empírica de cómo la piensan y la operan sus principales actores, docentes y gestores. Para conocer la percepción de estos dos actores el Proyecto "Determinantes y Orientaciones de la Calidad de la Educación Superior en América Latina y España” (DOCALE), realizó una investigación en universidades públicas de España, Argentina y México sobre la concepción de la calidad que consideraban más cercana quienes eran docentes, y la que percibían era la que prevalecía en quienes eran gestores en sus instituciones (Barandiarán, et al., 2011). Los conceptos de calidad que se utilizaron se construyeron sobre la base de la propuesta de Harvey y Green (1993) y fueron los siguientes:

- La calidad consiste en desarrollar las capacidades del estudiante para avanzar en su propia transformación.

- La calidad consiste en formar estudiantes capaces de asumir un compromiso social.

- La calidad consiste en el cumplimiento de los objetivos y la misión que ha establecido la institución.

- La calidad consiste en satisfacer las expectativas de todos los involucrados (alumnos, profesores, instituciones, sociedad).

- La calidad consiste en satisfacer las expectativas de los alumnos y de las organizaciones donde se colocan los egresados. 
- La calidad consiste en el cumplimiento y la mejora de estándares establecidos.

- La calidad consiste en conseguir la eficiencia: obtener los mejores resultados al menor costo.

Estas concepciones, como se ilustra a continuación, fueron agrupadas como "tradicionales" o más cercanos a las concepciones industriales y estandarizadas de la calidad propuestas por el enfoque de la Calidad Total, consistentes en alcanzar estándares y la excelencia, o la ganancia monetaria; o como "modernas" al estar más cercanas a los procesos de transformación humana.

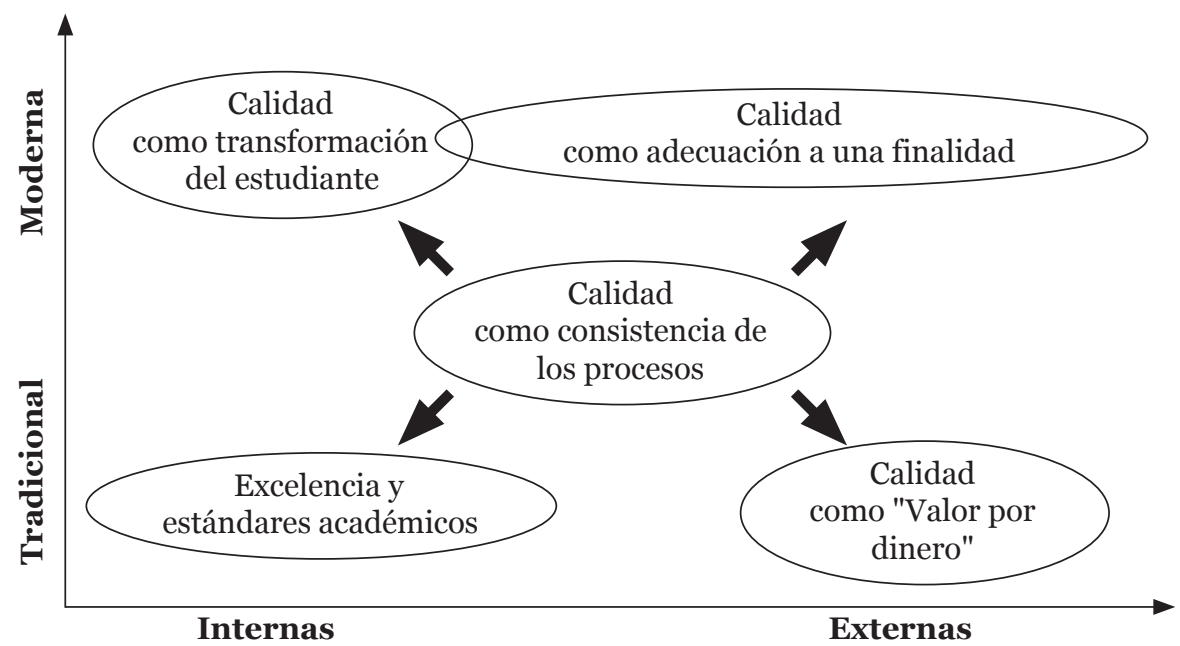

Figura1. Conceptos de calidad de la educación superior. Proyecto "Determinantes y Orientaciones de la Calidad de la Educación Superior en América Latina y España” (DOCALE). Fuente: Elaboración propia, equipo Red ECUALE (Estudios sobre la Calidad en las Universidades de América Latina y España)

La adhesión de las y los docentes mexicanos fue muy amplia para los conceptos "modernos" de calidad, en tanto que la de directivos y gestores lo fue para los conceptos "tradicionales" de calidad. Lo anterior revela, como evidencia empírica, que las concepciones de calidad que tienen estos dos actores y por tanto las acciones que estas percepciones se derivan, apuntan de manera divergente hacia la consecución de una buena educación.

En esta investigación se encontró también que los factores que los docentes consideran, entre otros, que son los que más influyen en una buena educación son: en la calidad de la educación, la sólida formación del profesorado y su motivación; 
en tanto que factores que son primordiales para quienes son gestores destacan, entre otros, la introducción y uso de tecnologías de la información y la comunicación, la acreditación y certificación; mientras que la internacionalización como desarrollo de espacios académicos supranacionales no tienen mucho impacto en la calidad de la docencia, pero son donde se destinan más recursos y esfuerzos quienes son directivos y gestores, lo que demuestra un serio conflicto de percepciones que sin duda influye en la realidad institucional y en los ambientes de trabajo que se generan.

Por tanto, las opciones de educación a distancia y, más específicamente, las opciones de educación virtual no tienen una alta valoración en la percepción que de la calidad educativa tienen quienes son docentes. Existen muchas explicaciones doctrinales al respecto, pero la evidencia empírica es aún insuficiente y hacia allá apuntaremos las siguientes investigaciones, para poder contar con elementos que ofrecer a quienes toman decisiones en este nivel educativo.

\section{¿CÓMO ASEGURAR QUE LA EDUCACIÓN A DISTANCIA SEA UNA BUENA EDUCACIÓN?}

Esta pregunta resume las preocupaciones y discusiones sobre la calidad en el servicio de la educación virtual, que como hemos sostenido, es válida para todo la educación en cualesquiera de sus modalidades. Por ello, para que la educación a distancia sea considerada una buena educación, una educación de calidad, además de la integración de la modalidad a distancia en el modelo educativo y académico, de desarrollar conocimiento autóctono para esta modalidad educativa, como para toda la educación, así como opciones tecnológicas propias, es necesario también reglamentar cómo se ofrece y se implementa la educación a distancia, en especial la educación virtual. El REVOE debe considerar los criterios de calidad para la educación presencial más los elementos propios de la educación a distancia, entre ellos, cuidar que las instituciones elijan la tecnología correcta. Asegurar ofrecer buenos programas es necesario, pero no suficiente, se requiere una estrategia de base amplia (broad-based learning strategy) que incluya: a) La construcción de una cultura de aprendizaje y el cambio en la forma de percibir la calidad del profesorado, directivos y gestores de manera que se armonicen y apunten hacia la misma dirección en los factores que influyen en la calidad de la docencia; b) anteponer la didáctica a los aspectos técnicos, cuidar, como ya se señaló, de no "tecnologizar la obsolescencia"; c) hacer sustentable el cambio del modelo de aprendizaje y asegurar su financiamiento, sustentable también. 


\section{REFERENCIAS BIBLIOGRÁFICAS}

Barandiarán Galdós, M.; Miren Barrenetxea A.; Cardona Rodríguez, A.; Martín López Armengol, A.; Mijangos del Campo, J.; y Olaskoaga Larrauri, J. (2011). Riqueza semántica de la calidad universitaria y debate sobre su gestión. Marco teórico de la investigación. En: López Armengol, M. (Coord.). Hacia una Educación Superior de Calidad (Volumen II): Una mirada de quienes gestionan las universidades en Argentina, España y México. Argentina, Editorial Universidad Nacional de La Plata (EDULP).

Bueno Macías, L.; Moreno Castañeda, M. (2000). Nuevos escenarios educativos. Memorias del VII encuentro internaiconal de Educación a distancia. México, Universidad de Guadalajara.

Consejo Nacional de Ciencia y Tecnología (2008). Programa Especial de Ciencia y Tecnología 2008-2012. Diario Oficial (Primera Sección), Martes 16 de diciembre. [en línea] Disponible en: www.conacyt.gob.mx/Convocatorias/ Varias/PECiTI.PDF (consulta 2011, 10 de abril).

Deming Edwards, W. (1989). Calidad, Productividad y Competitividad. La Salida de la Crisis. México, Díaz de Santos.

Didrikkson, A. (2003). La Universidad diferente: presente y futuro de la Universidad de América Latina y el Caribe. En: Inayatullah, Sohail y Jennifer Gideley (Comp). La Universidad en transformación. Perspectivas globales sobre los futuros de la Universidad. Barcelona, Pomares.

Fernández Lamarra, N. (2007). Educación superior y calidad en América Latina y Argentina. Los procesos de evaluación y acreditación. Buenos Aires, Argentina, IESALC/UNESCO-EDUNTREF.

Fernández Lamarra, N. (2010). Hacia una nueva agenda de la educación superior en América Latina. Situación y perspectivas. México, Temas de Hoy Número 028, ANUIES.

García, D.; Farías, F.; López, A. (2006). Reglamentode Instituciones y Programas de Educación Superior a Distancia. Santo Domingo, República Dominicana, Secretaría de Educación Superior, Ciencia y Tecnología (SEESCYT). [en línea]. Disponible en: http://www. seminariosined.ipn.mx/pagina/ documentos/REGLAMENTO DE EDUCACION A DISTANCIA RD.pdf. (consulta 2008, 18 de febrero).

Gayol, Y.; Seidensticker, L. (2004). Capacitación-e y a distancia para la gestión pública, la participación ciudadana y el desarrollo sostenible en las municipalidades de América Latina y el Caribe: hoja de ruta (Roadmap). $I X$ Congreso Internacional del CLAD sobre la Reforma del Estado y de la Administración Pública, Madrid, España, 2 - 5 Nov.

Harvey, L.; Green, D. (1993). Defining quality. Assessment and Evaluation in Higher Education, 18 (1).

Inayatullah, S.; Gideley, J. (Comp.) (2003). La Universidad en transformación. Perspectivas globales sobre los futuros de la Universidad. Barcelona, Pomares.

Latapí Sarre, P. (2008). Una buena educación. Reflexiones sobre la calidad. México, Universidad de Colima.

López Armengol, M. (Coord.) (2011). Hacia una Educación Superior de Calidad (Volumen II): Una mirada de quienes gestionan las universidades en Argentina, España y México. Argentina, Editorial Universidad Nacional de La Plata (EDULP).

Moreno Castañeda, M. et al., (2000). Desarrollo de ambientes de aprendizaje en educación a distancia. Textos del VI Encuentro Internacional de Educación 
a Distancia. México, Universidad de Guadalajara.

Moreno Castañeda, M. (2007). La calidad de la Educación a Distancia en ambientes virtuales. Apertura, noviembre, año/ volumen7, número oo6. México, Universidad de Guadalajara.

Nava Rogel,R.; MercadoSalgado,P.; Demuner Flores, M. (2010). Realidad, mitos y oportunidades de las universidades del conocimiento. México, SINNCO.

Neave, G. (2009). Studying Governance in Higher Education: Evacuating, Reconstructing and Re-engineering Meaning. Seminario UAM-A, México D.F. Septiembre.

OECD (2005). Education at a Glace. Paris, OCDE.

OCDE (2010). Panorama de la educación 2010: Indicadores de la OCDE España, Santillana.

Olivé, L.; Sandoval, R. (2007). Hacia la sociedad del conocimiento en México. Desafíos y perspectivas. Ciencia $y$ Desarrollo, Volumen 33, Número 211, Septiembre. México, CONACYT.

Peters, O. ( 2002 ). La educación a distancia en transición. Nuevas tendencias y retos.
México, Universidad de Guadalajara.

Turnbull, J. (2009). 9 habits of highly effective teachers a practical guide to empowerment. USA, Continuum.

UNESCO (2005). Hacia las sociedades del conocimiento. Informe Mundial, Paris, UNESCO [en línea]. Disponible en: http://www.iesalc. unesco.org.ve/pruebaobservatorio/ documentos\%20pdf/conocimiento.pdf (consulta 2011, 10 de abril).

UNESCO (2009). Conferencia Mundial de Educación Superior."La Nueva Dinámica de la Educación Superior y la búsqueda del cambio social y el Desarrollo". Comunicado Final. Paris, Francia, 05 - 08 de Julio.

UNESCO (1994). International Comission on Education for the Twenty-first Century. París, UNESCO.

World Bank (2002). Constructing knowledge societies: New challenges for tertiary education. Washington. [en línea]. Disponible en: http://www1.worldbank. org/education/tertiary/documents/Co nstructing\%20Knowledge\%20Societies. pdf (consulta 2011, 10 de abril).

\section{PERFIL ACADÉMICO Y PROFESIONAL DE LA AUTORA}

Elia Marúm-Espinosa. Directora y profesora investigadora del Centro para la Calidad e Innovación de la Educación Superior del Centro Universitario de Ciencias Económico-Administrativas de la Universidad de Guadalajara. Es miembro del Sistema Nacional de Investigadores y Coordinadora de la Cátedra UNESCO “Género, liderazgo y equidad".

Es Doctora en Economía por la Universidad Nacional Autónoma de México (UNAM). Maestra en Economía por el Centro de Investigación y Docencia Económicas (CIDE) y Licenciada en Economía por la Universidad Veracruzana. Es Profesora investigadora y Directora del Centro para la Calidad e Innovación de la Educación Superior (CCIES), del Centro Universitario de Ciencias Económico- 
Administrativas de la Universidad de Guadalajara. Miembro del Sistema Nacional de Investigadores (SNI), Coordinadora de la Cátedra UNESCO Género, Liderazgo y Equidad y Presidenta de la Red de Académicos de Iberoamérica, A.C.

E-mail: eliamarume@yahoo.com.mx

\section{DIRECCIÓN DE LA AUTORA:}

Periférico Norte 799 Edificio B-307, Núcleo Universitario Los Belenes

Zapopan, Jal., México.

C.P. 44100

Fechas de recepción del artículo: 06/07/11

Fecha de aceptación del artículo: 01/09/11

\section{Como citar este artículo:}

Marúm-Espinosa, E. (2011). Calidad en el servicio en la Educación a Distancia. Una perspectiva desde México. RIED. Revista Iberoamericana de Educación a Distancia, volumen 14, $\mathrm{n}^{\mathrm{O}}$ 2, pp. 49-62. 